\title{
Asymmetric organocatalytic decarboxylative Mannich reaction using $\beta$-keto acids: $A$ new protocol for the synthesis of chiral $\beta$-amino ketones
}

Chunhui Jiang, Fangrui Zhong and Yixin Lu*

\section{Letter}

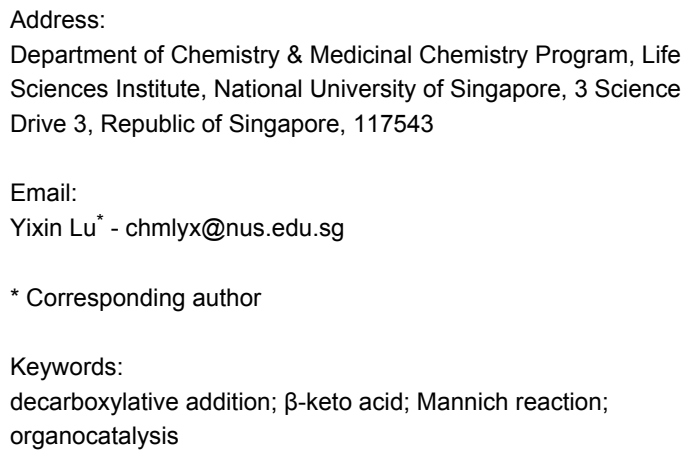

Department of Chemistry \& Medicinal Chemistry Program, Life Sciences Institute, National University of Singapore, 3 Science Drive 3, Republic of Singapore, 117543

Email:

Yixin Lu* - chmlyx@nus.edu.sg

* Corresponding author

Keywords:

decarboxylative addition; $\beta$-keto acid; Mannich reaction; organocatalysis

\author{
Beilstein J. Org. Chem. 2012, 8, 1279-1283. \\ doi:10.3762/bjoc. 8.144 \\ Received: 23 May 2012 \\ Accepted: 16 July 2012 \\ Published: 13 August 2012 \\ This article is part of the Thematic Series "Organocatalysis". \\ Guest Editor: B. List \\ (C) 2012 Jiang et al; licensee Beilstein-Institut. \\ License and terms: see end of document.
}

\begin{abstract}
The first decarboxylative Mannich reaction employing $\beta$-keto acids, catalyzed by cinchonine-derived bifunctional thiourea catalyst has been described. The desired $\beta$-amino ketones were obtained in excellent yields and with moderate to good enantioselectivities.
\end{abstract}

\section{Introduction}

Chiral $\beta$-amino ketones are an important class of building blocks for the synthesis of 1,3-amino alcohols [1,2], 1,3-amino acids [3] and other bioactive nature products [4-6]. Given their synthetic significance, methods for the asymmetric synthesis of $\beta$-amino ketones have been extensively investigated over the past few decades [7]. Among them, the Mukaiyama-Mannich reaction performed with silyl enol ethers and sulfonyl aldimines, catalyzed by a chiral Lewis acid complex, is one of the most important synthetic methods [8-13]. Apparently, direct use of inactivated ketones as a donor would be of great practical value. Indeed, direct approaches such as asymmetric enamine catalysis [14-17] and Brønsted acid catalysis [18] have been reported, through the activation of ketones or aryl imines [19]. However, substrates for the enamine activation are limited to only acetone and cyclic alkyl ketones. Application of aryl methyl ketones in the asymmetric Mannich reaction by enamine catalysis remains elusive. On the other hand, the only chiral Brønsted acid catalytic system based on BINOL-phosphates was reported by Rueping et al. Unfortunately, the yields of the reported reactions were unsatisfactory and the enantioselectivities were modest [20].

In recent years, inspired by the enzymatic synthesis of polyketides and fatty acids in biological systems, the enantioselective decarboxylative reactions of malonic acid half thioesters (MAHTs) have received much attention. In this regard, various electrophiles, including aldehydes, ketones, imines, activated alkenes and azodicarboxylates, have been employed as elec- 


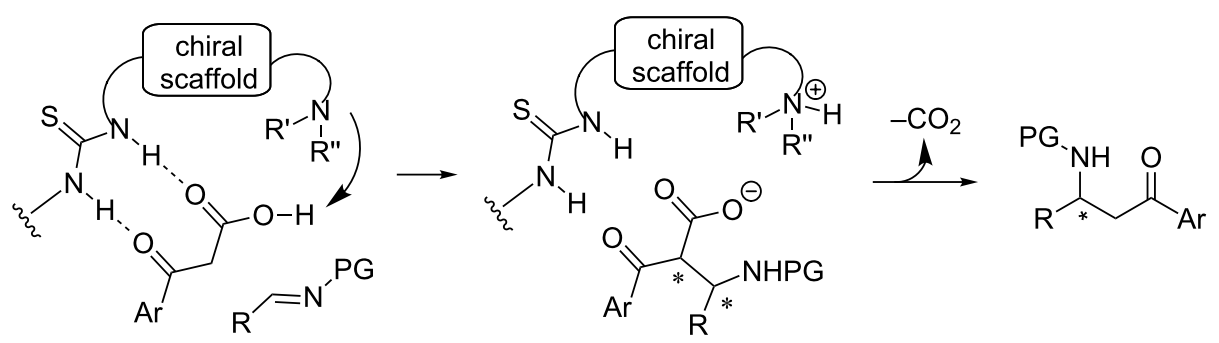

Scheme 1: Working hypothesis: Decarboxylative Mannich reaction.

trophiles in the presence of metal [21-25] or organocatalysts [26-36].

To provide a practical solution to the low reactivity associated with aryl methyl ketones, we wondered whether $\beta$-keto acids could serve as an enolate equivalent to aryl methyl ketones upon decarboxylation (Scheme 1). The proposed addition-decarboxylation sequence is consistent with current mechanistic understanding [31,35,37,38]. However, we cannot exclude an alternative decarboxylation-addition pathway at this stage. In fact, in sharp contrast to the popular use of malonic acid half thioesters (MAHTs) as an ester enolate equivalent in enantioselective decarboxylative additions [39], the employment of $\beta$-keto acids as a reaction partner in decarboxylative processes has rarely been explored [37]. Herein, we reported the first decarboxylative Mannich reaction between the $\beta$-keto acids and sulfonylimines, affording chiral $\beta$-amino ketones in excellent yields and good enantioselectivities.

\section{Findings}

In our initial screening, we examined the model reaction between tosylimine 1a and $\beta$-keto acid $\mathbf{2 a}$ in the presence of a range of bifunctional catalysts (Table 1). We first evaluated the catalytic effects of several cinchona alkaloid derivatives. Commercially available cinchonidine (CD-1) led to the formation of the product with disappointing enantioselectivity (Table 1, entry 1). Quinine-derived sulfonamide [40], $\beta$-isocupreidine ( $\beta$-ICD) [41,42] and biscinchona alkaloid (DHQ) 2 AQN were all found to be poor catalysts (Table 1, entries 2-4). On the other hand, cinchona alkaloid derived bifunctional thiourea tertiary amine catalysts afforded much improved results (Table 1, entries 5-7). Among them, the cinchonine based thiourea $\mathbf{C - 1}$ turned out to be the best catalyst, and the Mannich product was isolated with $58 \%$ ee (Table 1, entry 7). In addition, we also examined several other bifunctional catalysts based on amino acids $[43,44]$, including threonine derived Thr-1 [45], and tryptophan based Trp-1 [46], as well as threonine incorporated multifunctional catalyst CD-3 [47]. However, no further improvement could be achieved
(Table 1, entries 8-10). The influence of different imines on the reaction was subsequently explored, and it was found that the electronic nature of the sulfonyl protective groups affected the enantioselectivity. While the employment of nosylimine $\mathbf{1 b}$ led to decreased enantioselectivity (Table 1, entry 11), replacement of tosylimine 1a with $N$-( $p$-methoxybenzenesulfonyl)imine 1c resulted in further improvement, and the product was obtained in $65 \%$ ee (Table 1 , entry 12 ). However, when ethoxycarbonylimine 1d was used, nearly racemic products were obtained, suggesting the importance of the sulfonyl group in the asymmetric induction (Table 1, entry 13). Less reactive imines, such as diphenylphosphinoylimine $\mathbf{1 e}$ and Cbz-imine 1f, proved to be unsuitable for the reaction (Table 1, entries 14 and 15).

A screening of the solvent effect was then followed, and the results are summarized in Table 2. In general, the reaction proceeded very well in common aprotic solvents, and excellent yields were consistently obtained (Table 2, entries 1-9). Enantioselectivity of the reaction varied, and diethyl ether was found to be the best solvent, furnishing the desired product with $72 \%$ ee. Employment of other etheric solvents, including methyl tert-butyl ether and dioxane, and lowering reaction temperature did not offer further improvement (Table 2, entries 10-12).

To establish the substrate scope, a number of sulfonylimines derived from aromatic aldehydes were employed as acceptors, and the results are summarized in Table 3. In general, the reaction worked well for imines with various substituents at different positions of the phenyl ring, including electron-withdrawing groups, electron-donating groups and halogen atoms, and excellent yields and moderate ee values were obtained (Table 3, entries 1-10). Heterocycles were well-tolerated, and good enantioselectivities were obtained with 2-furyl and thiophen-2-yl containing substrates (Table 3, entries 11 and 12). The aryl groups of $\beta$-keto acids could also be varied, and the reaction was applicable to $\beta$-keto acids with different aromatic substituents (Table 3, entries 13-17). Furthermore, the reaction 
Table 1: Exploration of the decarboxylative addition of $\beta$-ketoacids to imines.

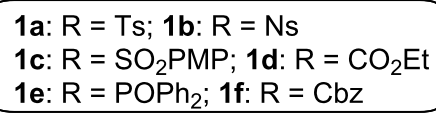

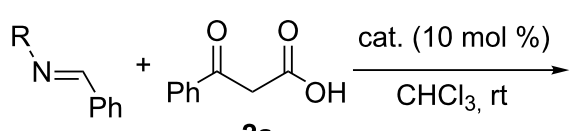

2a<smiles>[R]N[C@H](CC(=O)c1ccccc1)c1ccccc1</smiles>

3a-3f<smiles>C=CC1CN2CCC1C2[C@H](O)c1ccnc2ccccc12</smiles>

CD-1<smiles>C=CC1CN2CCC1C([C@H](NS(=O)(=O)O)c1ccnc3ccc(OC)cc13)C2</smiles><smiles>CCC12CN3CCN(C1)C(C3)C2c1ccnc2ccc(O)cc12</smiles><smiles>C=CC1CN2CCC1C2[C@H](NC(=S)NC(=O)OCc1ccccc1)c1ccnc2ccccc12</smiles><smiles>C=CC1CN2CCC1C2[C@H](NC(=S)NC1CCCCC1)c1ccnc2ccc(OC)cc12</smiles><smiles>C=CC1CN2CCC1C2[C@H](NC(=S)N[Al])c1ccnc2ccccc12</smiles><smiles>CC(O[R15](C)(C)C)C(NC(=S)N[Al])C(=O)NC1CC2CCN1CC2</smiles>

Thr-1 $\mathrm{Ar}=3,5-\mathrm{CF}_{3}-\mathrm{Ph}$<smiles>CCN(CC)C[C@H](Cc1c[nH]c2ccccc12)NC(=S)N[Al]</smiles><smiles>C=CC1CN2CCC1CN2C(NC(=O)C(NC(=S)NC1CCCCC1)C(C)C(F)(F)F)c1ccnc2ccccc12</smiles>

\begin{tabular}{|c|c|c|c|c|}
\hline Entry ${ }^{a}$ & 1 & cat & Yield $(\%)^{b}$ & ee $(\%)^{\mathrm{c}}$ \\
\hline 1 & $1 a$ & CD-1 & 86 & 17 \\
\hline 2 & $1 a$ & Q-1 & 54 & 13 \\
\hline 3 & $1 a$ & $(\mathrm{DHQ})_{2} \mathrm{AQN}$ & 90 & 2 \\
\hline 4 & $1 a$ & $\beta-I C D$ & 93 & 27 \\
\hline 5 & $1 a$ & CD-2 & 97 & 44 \\
\hline 6 & $1 a$ & QD-1 & 93 & 54 \\
\hline 7 & $1 a$ & $C-1$ & 95 & 58 \\
\hline 8 & $1 a$ & Thr-1 & 88 & 12 \\
\hline 9 & $1 a$ & Trp-1 & 95 & 21 \\
\hline 10 & $1 a$ & CD-3 & 92 & 13 \\
\hline 11 & $1 b$ & C-1 & 95 & 49 \\
\hline 12 & 1c & C-1 & 96 & 65 \\
\hline 13 & $1 d$ & $C-1$ & 91 & 5 \\
\hline 14 & $1 e$ & C-1 & trace & - \\
\hline 15 & $1 f$ & $C-1$ & trace & - \\
\hline
\end{tabular}

aReactions were performed with $1(0.05 \mathrm{mmol}), \mathbf{2 a}(0.075 \mathrm{mmol})$ and the catalyst $(0.005 \mathrm{mmol})$ in $\mathrm{CHCl}_{3}(0.5 \mathrm{~mL}) .{ }^{\mathrm{b}}$ lsolated yield. ${ }^{\mathrm{C}}$ Determined by HPLC analysis on a chiral stationary phase.

was also applicable to alkyl $\beta$-keto acids, and comparable chemical yields and enantioselectivities were attainable (Table 3 , entries 18-19). The absolute configurations of the products were assigned by comparing the optical rotation of $\mathbf{3 a}$ with the value reported in the literature [48] (see the Supporting Information File 1 for details).
In conclusion, we have developed the first organocatalytic decarboxylative Mannich reaction employing $\beta$-keto acids as the donor. The reaction was effectively catalyzed by cinchoninebased bifunctional catalyst $\mathbf{C - 1}$, and the synthetically useful $\beta$-amino ketones were prepared in excellent yields and with moderate to good enantioselectivities. The method reported 


\begin{tabular}{|c|c|c|c|}
\hline \multicolumn{2}{|c|}{$\mathrm{PMP}_{1 \mathrm{c}}^{1 \mathrm{c}}=\underset{2 \mathrm{a}}{\mathrm{2a}}$} & \multicolumn{2}{|c|}{$\stackrel{\substack{\mathrm{C}-1 \\
\text { st, } 12 \mathrm{~h}}}{\stackrel{\text { (10 mol \%) }}{\mathrm{PMPO}_{2} \mathrm{~S}_{-}}}$} \\
\hline Entry $^{a}$ & Solvent & Yield $(\%)^{b}$ & ee $(\%)^{c}$ \\
\hline 1 & $\mathrm{CHCl}_{3}$ & 96 & 65 \\
\hline 2 & THF & 93 & 64 \\
\hline 3 & DCM & 92 & 66 \\
\hline 4 & toluene & 90 & 63 \\
\hline 5 & diethyl ether & 93 & 72 \\
\hline 6 & ethyl acetate & 92 & 66 \\
\hline 7 & benzene & 90 & 67 \\
\hline 8 & DCE & 91 & 66 \\
\hline 9 & acetone & 92 & 52 \\
\hline 10 & methyl tert-butyl ethe & 92 & 62 \\
\hline 11 & dioxane & 94 & 65 \\
\hline $12^{d}$ & diethyl ether & 67 & 65 \\
\hline
\end{tabular}

${ }^{a}$ Reactions were performed with $1 c(0.05 \mathrm{mmol}), 2 \mathrm{2a}(0.075 \mathrm{mmol})$ and C-1 $(0.005 \mathrm{mmol})$ in the solvent specified $(0.5 \mathrm{~mL})$. blsolated yield. cDetermined by HPLC analysis on a chiral stationary phase. ${ }^{\mathrm{d}}$ Reaction was performed at $0{ }^{\circ} \mathrm{C}$.

Table 3: Substrate scope.

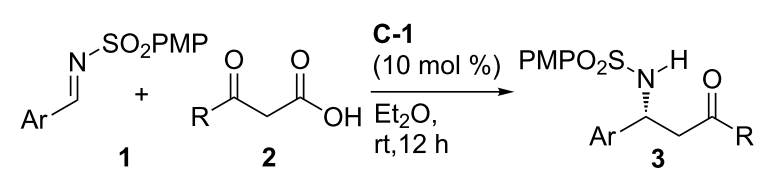

$\mathrm{PMP}=p$-methoxyphenyl

\begin{tabular}{|c|c|c|c|c|c|}
\hline Entry $^{a}$ & $\mathrm{Ar}$ & $\mathrm{R}$ & 3 & $\begin{array}{l}\text { Yield } \\
(\%)^{b}\end{array}$ & $\begin{array}{l}\text { ee } \\
(\%)^{c}\end{array}$ \\
\hline 1 & $\mathrm{Ph}$ & $\mathrm{Ph}$ & $3 c$ & 93 & 72 \\
\hline 2 & $4-\mathrm{Me}-\mathrm{C}_{6} \mathrm{H}_{4}$ & $\mathrm{Ph}$ & $3 g$ & 90 & 64 \\
\hline 3 & $4-\mathrm{Br}-\mathrm{C}_{6} \mathrm{H}_{4}$ & $\mathrm{Ph}$ & $3 h$ & 88 & 61 \\
\hline 4 & $4-\mathrm{CF}_{3}-\mathrm{C}_{6} \mathrm{H}_{4}$ & $\mathrm{Ph}$ & $3 \mathbf{i}$ & 85 & 55 \\
\hline 5 & $4-\mathrm{OMe}-\mathrm{C}_{6} \mathrm{H}_{4}$ & $\mathrm{Ph}$ & $3 \mathbf{j}$ & 97 & 62 \\
\hline 6 & $2-\mathrm{F}-\mathrm{C}_{6} \mathrm{H}_{4}$ & $\mathrm{Ph}$ & $3 k$ & 89 & 65 \\
\hline 7 & $2-\mathrm{Me}-\mathrm{C}_{6} \mathrm{H}_{4}$ & $\mathrm{Ph}$ & 31 & 92 & 65 \\
\hline 8 & $2-\mathrm{Br}-\mathrm{C}_{6} \mathrm{H}_{4}$ & $\mathrm{Ph}$ & $3 m$ & 87 & 59 \\
\hline 9 & $3-\mathrm{Me}-\mathrm{C}_{6} \mathrm{H}_{4}$ & $\mathrm{Ph}$ & $3 n$ & 97 & 65 \\
\hline 10 & $3-\mathrm{Br}-\mathrm{C}_{6} \mathrm{H}_{4}$ & $\mathrm{Ph}$ & 30 & 96 & 61 \\
\hline 11 & 2-furyl & $\mathrm{Ph}$ & $3 p$ & 94 & 83 \\
\hline 12 & thiophen-2-yl & $\mathrm{Ph}$ & $3 q$ & 87 & 77 \\
\hline 13 & $\mathrm{Ph}$ & $4-\mathrm{F}-\mathrm{C}_{6} \mathrm{H}_{4}$ & $3 r$ & 95 & 64 \\
\hline $14^{\mathrm{d}}$ & $\mathrm{Ph}$ & $3-\mathrm{Cl}-\mathrm{C}_{6} \mathrm{H}_{4}$ & $3 s$ & 62 & 70 \\
\hline 15 & $\mathrm{Ph}$ & 2-naphthyl & $3 t$ & 62 & 69 \\
\hline 16 & $\mathrm{Ph}$ & $4-\mathrm{Me}-\mathrm{C}_{6} \mathrm{H}_{4}$ & $3 u$ & 93 & 67 \\
\hline 17 & $\mathrm{Ph}$ & $2-\mathrm{OMe}-\mathrm{C}_{6} \mathrm{H}_{4}$ & $3 v$ & 88 & 60 \\
\hline
\end{tabular}

Table 3: Substrate scope. (continued)

\begin{tabular}{llllll}
18 & $\mathrm{Ph}$ & $n-\mathrm{Pr}$ & $3 \mathbf{w}$ & 92 & 54 \\
19 & $\mathrm{Ph}$ & $t-\mathrm{Bu}$ & $3 \mathbf{x}$ & 75 & 73 \\
\hline
\end{tabular}

aaeactions were performed with $1(0.05 \mathrm{mmol}), 2(0.075 \mathrm{mmol})$ and C-1 $(0.005 \mathrm{mmol})$ in $\mathrm{Et}_{2} \mathrm{O}(0.5 \mathrm{~mL})$. ${ }^{\mathrm{b}}$ /solated yield. ${ }^{\text {CDetermined by }}$ HPLC analysis on a chiral stationary phase. ${ }^{\mathrm{d}}$ The catalyst loading was $20 \mathrm{~mol} \%$.

represents a new protocol for the asymmetric construction of $\beta$-amino ketones.

\section{Experimental}

General procedure for the decarboxylative Mannich reaction of $\beta$-keto acids and aldimines

To a solution of imine $1 \mathrm{c}(13.8 \mathrm{mg}, 0.05 \mathrm{mmol})$ and $\mathbf{C}-\mathbf{1}$ $(2.8 \mathrm{mg}, 0.005 \mathrm{mmol})$ in ether $(0.5 \mathrm{~mL})$ at room temperature, was added $\beta$-keto acid $2 \mathrm{a}(12.3 \mathrm{mg}, 0.075 \mathrm{mmol})$. The reaction mixture was stirred for $12 \mathrm{~h}$. The solvent was then removed under reduced pressure, and the residue was purified by flash chromatography on silica gel (hexane/ethyl acetate $5: 1$ to $3: 1$ ) to afford $\mathbf{3 c}$ as a white solid (18.4 $\mathrm{mg}, 93 \%$ yield).

\section{Supporting Information}

\section{Supporting Information File 1}

Characterization data and spectra of synthesized compounds.

[http://www.beilstein-journals.org/bjoc/content/ supplementary/1860-5397-8-144-S1.pdf]

\section{Acknowledgements}

We are grateful for generous financial support from the National University of Singapore (R-143-000-469-112) and GSK-EDB (R-143-000-491-592).

\section{References}

1. Barluenga, J.; Viado, A. L.; Aguilar, E.; Fustero, S.; Olano, B. J. Org. Chem. 1993, 58, 5972-5975. doi:10.1021/jo00074a024

2. Enders, D.; Moser, M.; Geibel, G.; Laufer, M. C. Synthesis 2004, 2040-2046. doi:10.1055/s-2004-829142

3. Mukhopadhyay, M.; Bhatia, B.; Iqbal, J. Tetrahedron Lett. 1997, 38, 1083-1086. doi:10.1016/S0040-4039(96)02474-4

4. Arend, M.; Westerman, B.; Risch, N. Angew. Chem., Int. Ed. 1998, 37, 1044-1070.

doi:10.1002/(SICI)1521-3773(19980504)37:8<1044::AID-ANIE1044>3. 0.CO;2-E

5. Evans, G. B.; Furneaux, R. H.; Tyler, P. C.; Schramm, V. L. Org. Lett. 2003, 5, 3639-3640. doi:10.1021/ol035293q 
6. Joshi, N. S.; Whitaker, L. R.; Francis, M. B. J. Am. Chem. Soc. 2004, 126, 15942-15943. doi:10.1021/ja0439017

7. Kobayashi, S.; Ishitani, H. Chem. Rev. 1999, 99, 1069-1094. doi:10.1021/cr980414z

8. Ishitani, H.; Ueno, M.; Kobayashi, S. J. Am. Chem. Soc. 1997, 119, 7153-7154. doi:10.1021/ja970498d

9. Kobayashi, S.; Ishitani, H.; Ueno, M. J. Am. Chem. Soc. 1998, 120, 431-432. doi:10.1021/ja973527t

10. Hagiwara, E.; Fujii, A.; Sodeoka, M. J. Am. Chem. Soc. 1998, 120, 2474-2475. doi:10.1021/ja973962n

11. Ishitani, H.; Ueno, M.; Kobayashi, S. J. Am. Chem. Soc. 2000, 122, 8180-8186. doi:10.1021/ja001642p

12. Juhl, K.; Gathergood, N.; Jørgensen, K. A. Angew. Chem., Int. Ed. 2001, 40, 2995-2997. doi:10.1002/1521-3773(20010817)40:16<2995::AID-ANIE2995>3.0.CO ;2-M

13. Taggi, A. E.; Hafez, A. E.; Lectka, T. Acc. Chem. Res. 2003, 36, 10-19. doi:10.1021/ar020137p

14. Notz, W.; Tanaka, F.; Barbas, C. F., III. Acc. Chem. Res. 2004, 37, 580-591. doi:10.1021/ar0300468

15. Mukherjee, S.; Yang, J. W.; Hoffmann, S.; List, B. Chem. Rev. 2007, 107, 5471-5569. doi:10.1021/cr0684016

16. Melchiorre, P.; Marigo, M.; Carlone, A.; Bartoli, G. Angew. Chem., Int. Ed. 2008, 47, 6138-6171. doi:10.1002/anie.200705523

17. Kano, T.; Maruoka, K. Chem. Commun. 2008, 5465-5473. doi:10.1039/B809301F

18. Akiyama, T. Chem. Rev. 2007, 107, 5744-5758. doi:10.1021/cr068374j

19. Verkade, J. M. M.; van Hemert, L. J. C.; Quaedflieg, P. J. L. M.; Rutjes, F. P. J. T. Chem. Soc. Rev. 2008, 37, 29-41. doi: $10.1039 / \mathrm{b} 713885 \mathrm{~g}$

20. Rueping, M.; Sugiono, E.; Schoepke, F. R. Synlett 2007, 1441-1445. doi:10.1055/s-2007-980369

21. Lalic, G.; Aloise, A. D.; Shair, M. D. J. Am. Chem. Soc. 2003, 125, 2852-2853. doi:10.1021/ja029452x

22. Orlandi, S.; Benaglia, M.; Cozzi, F. Tetrahedron Lett. 2004, 45, 1747-1749. doi:10.1016/j.tetlet.2003.12.089

23. Magdziak, D.; Lalic, G.; Lee, H. M.; Fortner, K. C.; Aloise, A. D.; Shair, M. D. J. Am. Chem. Soc. 2005, 127, 7284-7285. doi:10.1021/ja051759j

24. Fortner, K. C.; Shair, M. D. J. Am. Chem. Soc. 2007, 129, 1032-1033. doi:10.1021/ja0673682

25. Furutachi, M.; Mouri, S.; Matsunaga, S.; Shibasaki, M. Chem.-Asian J. 2010, 5, 2351-2354. doi:10.1002/asia.201000540

26. Brunner, H.; Müller, J.; Spitzer, J. Monatsh. Chem. 1996, 127, 845-858. doi:10.1007/BF00807023

27. Ryu, Y.; Scott, A. I. Tetrahedron Lett. 2003, 44, 7499-7502. doi:10.1016/j.tetlet.2003.08.014

28. List, B.; Doehring, A.; Fonseca, M. T. H.; Wobser, K.; van Thienen, H.; Torres, R. R.; Galilea, P. Adv. Synth. Catal. 2005, 347, 1558-1560. doi:10.1002/adsc.200505196

29. Blanchet, J.; Baudoux, J.; Amere, M.; Lasne, M.-C.; Rouden, J. Eur. J. Org. Chem. 2008, 5493-5506. doi:10.1002/ejoc.200800759

30. Blaquiere, N.; Shore, D. G.; Rousseaux, S.; Fagnou, K. J. Org. Chem. 2009, 74, 6190-6198. doi:10.1021/jo901022j

31. Ricci, A.; Pettersen, D.; Bernardi, L.; Fini, F.; Fochi, M.; Herrera, R. P.; Sgarzani, V. Adv. Synth. Catal. 2007, 349, 1037-1040. doi:10.1002/adsc.200600536

32. Lubkoll, J.; Wennemers, H. Angew. Chem., Int. Ed. 2007, 46, 6841-6844. doi:10.1002/anie.200702187
33. Pan, Y.; Kee, C. W.; Jiang, Z.; Ma, T.; Zhao, Y.; Yang, Y.; Xue, H.; Tan, C.-H. Chem.-Eur. J. 2011, 17, 8363-8370. doi:10.1002/chem.201100687

34. Bae, H. Y.; Some, S.; Lee, J. H.; Kim, J.-Y.; Song, M. J.; Lee, S.; Zhang, Y. J.; Song, C. E. Adv. Synth. Catal. 2011, 353, 3196-3202. doi:10.1002/adsc.201100458

35. Baudoux, J.; Lefebvre, P.; Legay, R.; Lasne, M.-C.; Rouden, J. Green Chem. 2010, 12, 252-259. doi:10.1039/b915681j

36. Hara, N.; Nakamura, S.; Funahashi, Y.; Shibata, N. Adv. Synth. Catal. 2011, 353, 2976-2980. doi:10.1002/adsc.201100410

37. Zheng, Y.; Xiong, H.-Y.; Nie, J.; Hua, M.-Q.; Ma, J.-A. Chem. Commun. 2012, 48, 4308-4310. doi:10.1039/c2cc30949a

38. Yang, C.-F.; Shen, C.; Wang, J.-Y.; Tian, S.-K. Org. Lett. 2012, 14, 3092-3095. doi:10.1021/ol301180z

39. Pan, Y.; Tan, C.-H. Synthesis 2011, 2044-2053. doi:10.1055/s-0030-1260607

40. Luo, J.; Xu, L.-W.; Hay, R. A. S.; Lu, Y. Org. Lett. 2009, 11, 437-440. doi:10.1021/ol802486m

41. Marcelli, T.; Maarseveen, J. H.; Huiemstra, H. Angew. Chem., Int. Ed. 2006, 45, 7496-7504. doi:10.1002/anie.200602318

42. Zhong, F.; Chen, G.-Y.; Lu, Y. Org. Lett. 2011, 13, 82-85. doi:10.1021/ol102597s

43. Xu, L.-W.; Lu, Y. Org. Biomol. Chem. 2008, 6, 2047-2053. doi:10.1039/b803116a

44. Xu, L.-W.; Luo, J.; Lu, Y. Chem. Commun. 2009, 1807-1821. doi:10.1039/b821070e

45. Dou, X.; Han, X.; Lu, Y. Chem.-Eur. J. 2012, 18, 85-89. doi:10.1002/chem.201102796

46. Han, X.; Kwiatkowski, J.; Xue, F.; Huang, K.-W.; Lu, Y. Angew. Chem., Int. Ed. 2009, 48, 7604-7607. doi:10.1002/anie.200903635

47. Zhu, Q.; Lu, Y. Angew. Chem., Int. Ed. 2010, 49, 7753-7756. doi:10.1002/anie.201003837

48. Zhao, C.-H.; Liu, L.; Wang, D.; Chen, Y.-J. Eur. J. Org. Chem. 2006, 2977-2986. doi:10.1002/ejoc.200600147

\section{License and Terms}

This is an Open Access article under the terms of the Creative Commons Attribution License (http://creativecommons.org/licenses/by/2.0), which permits unrestricted use, distribution, and reproduction in any medium, provided the original work is properly cited.

The license is subject to the Beilstein Journal of Organic Chemistry terms and conditions: (http://www.beilstein-journals.org/bjoc)

The definitive version of this article is the electronic one which can be found at: $\underline{\text { doi: } 10.3762 / \text { bjoc. } 8.144}$ 